\title{
Journal of Membrane Biology: Biotechnology and Engineering Section
}

\author{
Stavroula Sofou ${ }^{1} \cdot$ Mark Borden $^{2}$
}

Published online: 18 April 2016

(C) Springer Science+Business Media New York 2016

We are excited to introduce a new section on Biotechnology and Engineering in the newly re-organized Journal of Membrane Biology (JMBi). With this initiative, we aim to create a focal point for studies reporting on the physicochemical (and on the (bio)physical and (bio)chemical) characterization of lipid membranes whose structure and properties are driven by the requirements set by specific biomedical applications. In particular, we invite studies on lipid membranes which emphasize the design 'rules', both experimental and computational, and the engineering decisions to control/optimize membrane properties as dictated by their biomedical applicability. This new Section is meant to address a current gap in the established journals, which tend to emphasize either the fundamental biophysical characterization of biological membranes or the evaluation of synthetic lipid membrane-containing constructs for biomedical and biotech applications.

We invite our colleagues to consider the new Section on Biotechnology and Engineering as the place to submit high-quality work that bridges fundamental lipid membrane studies and applied in vivo evaluations. We welcome contributions on the study of lipid membranes in the form of bilayers, monolayers, and supported membranes. Examples include, but are not limited to, the following:

- Lipid membrane-containing materials, such as vesicles (liposomes), microbubbles, emulsions, foams, aerosols, gels, lipoplexes, and nanoparticles.

- Lipid membrane-containing architectures, such as supported membranes, Langmuir-Blodgett films, Newton black films, layer-by-layer films, membranes spanning microfluidic channels, and other possibilities.

- Lipid constructs designed for specific biomedical applications, such as imaging, drug and gene delivery, theranostics, and biosensing.

This section focuses exclusively on lipid membranes, and thereby provides a venue for researchers with common biological, physicochemical, and biomedical interests in lipid membranes. We are looking forward to our colleagues' responses and suggestions; our hope is that this initiative will result in the creation of an interacting and productive community.

Stavroula Sofou

ss1763@ rci.rutgers.edu

1 Department of Biomedical Engineering Rutgers, The State University of New Jersey, 599 Taylor Road, Piscataway, NJ 08854, USA

2 University of Colorado-Boulder, Boulder, CO, USA 